\title{
The pathogenesis of atherosclerosis
}

\author{
R.G. G errity, A.S. A ntonov \\ Department of Pathology, Medical College of Georgia, Augusta, Georgia, USA
}

Atherosclerosis is the underlying cause of arterial occlusion resulting in myocardial and cerebral infarction, and is the leading cause of death in Western Europe and the United States [1]. It is estimated that in the United States alone, about six million persons have symptomatic myocardial ischaemia, and that approximately one American dies every minute because of atherosclerotic coronary artery disease. Furthermore, atherosclerosis is the major complication of diabetes mellitus, and several studies indicate that diabetic patients have 2-5 times the death rate due to atherosclerotic disease than non-diabetic patients [2]. The relative risk of peripheral vascular disease in diabetic patients is even greater. In the past two decades, atherosclerosis has been the subject of intense study into both its causative (risk) factors as well as the pathobiology of the lesions. Although epidemiologically derived risk factors can be statistically correlated with clinical disease, it has proven difficult to correlate them with lesion pathology. The extremely long course of the disease prior to clinical significance, together with the impossibility of sequentially sampling human specimens, has made it difficult to conclusively identify the sequence of human plaque development. As a result, much of our understanding of the cellular pathobiology of atherosclerosis has been obtained from animal models and from tissue culture of cells isolated from the arterial wall and blood. Although no single animal model reproduces the human disease exactly, such models have been invaluable in furthering our understanding of the cellular composition, biochemistry, localization and structure of plaques. The pathogenesis of diabetic atherosclerosis is even more poorly understood. There is no evidence to suggest that the pathomorphology of the disease, that is, the

Corresponding author: Dr. R. G. Gerrity, Department of Pathology, Medical College of Georgia, Augusta, GA 309123605, USA

A bbreviations: PDGF, Platelet derived growth factor; LDL, low density lipoprotein sequence of plaque types and plaque composition, is different in diabetic patients, only that the progression of the disease is grossly accelerated. If this is the case, one approach to the problem is to identify factors and mechanisms in diabetic conditions which accelerate the atherogenic mechanisms known to occur in nondiabetic states. However, this approach as been hampered by the lack of good humanoid animal models of diabetic atherosclerosis.

The lesions of atherosclerosis, or plaques, have generally been classified in three categories: the fatty streak, the fibrous plaque, and the complicated lesion $[3,4]$. Of these lesion types, the fatty streak is the earliest to be seen in both humans and hyperlipaemic animal models. In humans, it is common in pre-teenage children, and by age 30 years, up to $30-50 \%$ of the aortic intimal surface may be covered by fatty streak lesions. Fatty streaks are grossly flat, lipid-rich lesions consisting predominantly of lipid-laden macrophages and minimal numbers of smooth muscle cells. The high lipid content of these lesions gives them a typical yellowish colour on gross examination. Such lesions do not occlude the lumen, and therefore do not present clinical symptoms. In contrast, the fibrous plaque is representative of various forms of advanced atherosclerosis and is widely accepted as the most common atherosclerotic precursor to occlusive lesions. As such, it is the major determinant of clinically significant disease. This lesion is whitish in gross appearance and protrudes into the vessel lumen. Its distribution is more focal in nature than that of fatty streaks. Histologically, fibrous plaques consist of layers of initial smooth muscle cells, many of which are lipid-laden, surrounded by collagen fibrils and elastin fragments in a proteoglycan-rich matrix forming a "fibrous cap" overlying a core of lipid-laden macrophages, extracellular lipid and necrotic cellular debris. Early fatty streaks do regress, but fibrous plaques in older age are found at the same anatomical locations as these early fatty streaks, particularly in the coronary and extracranial cerebral arteries. 
Virtually all occlusive lesions are of the third type, the complicated lesion, resulting from necrosis, calcification, and mural thrombosis of fibrous plaques. Although there is good evidence that fibrous plaques are complicated by these phenomena, and that plaque rupture and thrombosis may occur episodically, thus adding to plaque volume and occlusion, the relationship between fatty streaks and fibrous plaques remains less clear and a matter of controversy. More recently, McGill [3] and others have proposed the existence of a transitional lesion which may be the link between the fatty streak and the fibrous plaque. Grossly, these lesions resemble fatty streaks, but microscopically contain a core of necrotic debris and extracellular lipid not seen in the fatty streak, thus displaying morphological characteristics of both fatty streak and fibrous plaque. Chronologically, these lesions occur predominantly in 20-30-year-old males, at sites where fatty streaks are found in younger men, and fibrous plaques are found in older males [3]. If this is correct, then the transitional lesion may represent a relatively short-lived lesion transitional between fatty streaks and fibrous plaques.

The events involved in the progression of atherosclerosis from fatty streak to fibrous plaque to clinically significant atherosclerosis may never be elucidated with certainty in humans. However, as stated above, analysis of this sequence in humanoid animal models [4-9] of atherosclerosis has proven to be particularly useful, and in many instances, identical or similar counterparts have been found in human vessels derived from both surgically removed and autopsy material. It is now universally accepted that areas of predilection to atherosclerosis exist in the arteries of both man and experimental animals, and that this predilection, or susceptibility, results from functional differences in the vessel wall at these sites, possibly induced by haemodynamic conditions [10]. Such lesion-susceptible areas have been studied extensively in swine $[4-6,10]$ and pigeon [11] models, and recent studies have shown them to exist in human arteries. Systematic studies of lesion-susceptible areas in animal models shows that these areas have characteristics of enhanced endothelial permeability and intimal accumulation of blood macromolecules even in normal animals [10]. In conditions of hyperlipaemia, the earliest detectable event is a preferential enhanced uptake and accumulation of atherogenic low-density lipoprotein (LDL) across an intact endothelium [12], probably through a mechanism of increased bulk phase vesicular transport [10]. Similar accumulation of LDL has been shown in arteries from young humans [13].

In these lesion-susceptible areas, the earliest discernible cellular event in atherogenesis is the adhesion of blood monocytes to the endothelium, and their migration into the intima through endothelial junctions [5-8]. This occurs without disruption of endothelial integrity, beginning after only a few weeks of hyperlipaemia, and at times concurrent with the accumulation of LDL in the intima [12]. Recruitment of monocytes into these lesion-susceptible sites is mediated by the production of chemotactic factors in these areas which are induced in hyperlipaemia [14]. More recent studies have also identified an array of endothelial adhesion molecules which are actively involved in the initial adhesion of monocytes and other leukocytes to the endothelial lining [15]. Both monocyte chemotactic factors and adhesion molecules have been shown to be inducible on endothelial cells in culture by oxidized and modified LDL [16].

Intimal monocytes thus recruited into the vessel wall are rapidly transformed into lipid-laden macrophage foam cells by the unregulated uptake of modified lipoproteins via the scavenger and oxidized LDL receptor pathways $[17,18]$. Their increase in size as they accumulate lipid is the major contributor to increased lesion volume during the development of fatty lesions. There is evidence from the swine model that large numbers of macrophages migrate back through the endothelium into the blood and are cleared by the reticuloendothelial system, particularly at the fatty streak stage [6]. The monocyte may thus serve as an early defence mechanism to clear excess lipid from the lesion. However, it is clear that as lesions progress, foam cell necrosis contributes to the lipid core of the plaque [19]. There is evidence from both non-human primate and swine models that endothelial cell damage and retraction occur at sites where foam cells migrate or are in close proximity to the endothelium, and that these serve as foci for platelet adherence [5-8]. Endothelial cell damage at these sites may be associated with excessive stretching of the endothelium by plaque growth, enhanced membrane fragility due to altered lipid composition, release of lytic enzymes by macrophages, or toxicity due to oxidation of intimal lipoproteins by the macrophages themselves [18]. Although the ability of macrophages to actively oxidize lipids may be a mechanism whereby they enhance lipoprotein uptake, it is also clear that lipoproteins oxidized by macrophages are toxic to endothelial and smooth muscle cells, and as such, macrophages may contribute to the necrotic processes within the plaque. Furthermore, activated macrophages have been shown to secrete growth factors for smooth muscle cells [9], including a mitogen similar to platelet derived growth factor (PDGF). Mitogens released by macrophages and by platelets adherent to sites of endothelial damage or retraction may induce proliferation of smooth muscle cells in the intima derived from either pre-existing intimal cell masses or from smooth muscle cells which have migrated from the media. PDGF may also take part in this latter process, as it is known to be chemotactic for smooth muscle cells. Smooth muscle cell 
proliferation may occur at different stages in different plaques, depending upon geographic site, the existence of intimal smooth muscle cell masses, or the proximity of medial cells to the intima. Regardless, these cells form a fibrous cap over the lipid core of the lesion and add to plaque growth and progression by increased production of collagen, elastin, and matrix components. The trapping of lipid-laden foam cells beneath the fibrous cap leads to their necrosis, contributing to the mass of extracellular lipid in the core of the lesion [19]. Protrusion of the plaque into the lumen, together with increased fragility of endothelial cell membranes may also result in episodic plaque rupture or endothelial denudation resulting in focal fibrin-platelet thrombi which may become incorporated into the plaque, increasing plaque volume. Growth of the fibrous plaque by cell proliferation, connective tissue synthesis and complicating sequelae of rupture and thrombosis ultimately result in clinically significant occlusion.

It is now accepted that these events occur in the atherogenic sequence, and that some are manifest earlier than others, at least in animal models. For instance, enhanced LDL deposition and monocyte recruitment can be detected even at short durations of hyperlipidaemia. This occurs both in predisposed sites destined to form fatty streaks, and those which form fibrous plaques, suggesting that the initial response may be of a protective, inflammatory nature. Other events may occur later, and the occurrence of macrophages, other leukocytes and smooth muscle in advanced plaques, together with mural thrombi suggest that all processes may ultimately be ongoing simultaneously or episodically. Although it is apparent that endothelial cells, monocytes, macrophages, and smooth muscle cells all undergo structural and functional changes at various stages, and all play a role in atherogenesis, our understanding of the in vivo interactions among the three cell types is incomplete. Recent research has focused on the molecular mechanisms controlling the interactions among the three cell types, and elucidation of such interactions is key to our understanding of the atherogenic sequence. Also critical to our understanding of atherogenesis is how risk factors linked to an increased incidence of atherosclerosis are translated at cellular and molecular levels to initiate or augment the mechanisms described. This knowledge is fundamental to the development of new approaches to intervention and prevention, as well as novel tools for early diagnosis.

\section{References}

1. Report of the Working Group of Arteriosclerosis of the National Heart, Lung, and Blood Institute (1981) DHEW No. (NIH) 82-2035, vol 2, US Government Printing Office, Washington, DC
2. Steiner G (1995) Diabetes and atherosclerosis: epidemiology and intervention trials. In: Woodford FP, Davignon J, Sniderman A (eds) Artherosclerosis X. Elsevier, Amsterdam, pp 749-752

3. McGill HC Jr (1990) Questions about the natural history of human atherosclerosis. In: Glagov S, Newman WP III, Schaffer SA (eds) Pathobiology of the human atherosclerotic plaque. Springer, Berlin Heidelberg New York, pp 1-11

4. Gerrity RG (1989) Morphological development of the atherosclerotic plaque. In: Subbiah MTR (ed) Atherosclerosis: a pediatric perspective. CRC Press, Boca Raton, pp 930

5. Gerrity RG (1981) The role of the monocyte in atherogenesis. I. Transition of blood-borne monocytes into foam cells in fatty lesions. Am J Pathol 103: 181-190

6. Gerrity RG (1981) The role of the monocyte in atherogenesis. II. Migration of foam cells from atherosclerotic lesions. Am J Pathol 103: 191-200

7. Faggiotto A, Ross R, Harker L (1984) Studies of hypercholesterolemia in the nonhuman primate. I. Changes that lead to fatty streak formation. Arteriosclerosis 4: 323-340

8. Faggiotto A, Ross R (1984) Studies of hypercholesterolemia in the nonhuman primate. II. Fatty streak conversion to fibrous plaque. Arteriosclerosis 4: 341-356

9. Ross R (1986) The pathogenesis of atherosclerosis - an update. N Engl J Med 314: 488-500

10. Gerrity RG (1990) Arterial endothelial structure and permeability as it relates to susceptibility to atherogenesis. In: Glagov S, Newman WP III, Schaffer SA (eds) Pathobiology of the human atherosclerotic plaque. Springer, Berlin Heidelberg New York, pp 13-45

11. Jerome WG, Lewis JC (1985) Early atherogenesis in White Carneau pigeons. II. Ultrastructural and cytochemical observations. Am J Pathol 119: 210-222

12. Feldman DL, Hoff HF, Gerrity RG (1984) Immunohistochemical localization of apoprotein B in aortas from hyperlipemic swine. Preferential accumulation in lesionprone areas. Arch Pathol Lab Med 108: 817-822

13. Hoff HF (1976) Apolipoprotein localization in human cranial arteries, coronary arteries and the aorta. Stroke 7: 390-393

14. Gerrity RG, Goss JA, Soby L (1985) Control of monocyte recruitment by chemotactic factor(s) in lesion-prone areas of swine aorta. Artenosclerosis 5: 55-66

15. Pober JS, Cotran RS (1990) Cytokine-endothelial interactions in inflammation, immunity, and vascular injury. J Am Soc Nephrol 1: 225-235

16. Cushing SD, Berliner JA, Valente AJ, et al. (1990) Minimally modified low density lipoprotein induces monocyte chemotactic protein 1 in human endothelial cells and smooth muscle cells. Proc Natl Acad Sci USA 87: 51345138

17. Mahley RW, Innerarity TL (1983) Lipoprotein receptors and cholesterol homeostasis. Biochim Biophys Acta 737: 197-222

18. Steinberg D (1995) The oxidative modification hypothesis of atherogenesis: strengths and weaknesses. In: Woodford FP, Davignon J, Sniderman A (eds) Atherosclerosis X. Elsevier, Amsterdam, pp 25-29

19. Stary HC (1990) Changes in the cells of atherosclerotic lesions as advanced lesions evolve in coronary arteries of children and young adults. In: Glagov S, Newman WP III, Schaffter SA (eds) Pathobiology of the human atherosclerotic plaque. Springer, Berlin Heidelberg New York, pp 93-106 